\title{
Measurement of the $W$ boson mass with the Dø detector and combination of the CDF and $D \varnothing$ results for the $W$ boson mass
}

\author{
Jan Stark ${ }^{* \dagger}$ \\ LPSC Grenoble, France \\ E-mail: starkein2p3.fr
}

\begin{abstract}
The experimental precision on the $W$ boson mass is currently the limiting factor in indirect constraints on the mass of the standard model Higgs boson. We present a new precision measurement of the $W$ boson mass using data corresponding to $4.3 \mathrm{fb}^{-1}$ of integrated luminosity collected with the DØ detector during Run II at the Fermilab Tevatron. We measure $M_{W}=80.367 \pm 0.026 \mathrm{GeV}$. The combination with an earlier $\mathrm{D} \emptyset$ result, obtained using an independent Run II data sample which corresponds to $1 \mathrm{fb}^{-1}$, yields the new DØ Run II result: $M_{W}=80.375 \pm 0.023 \mathrm{GeV}$. We further present the new world average for the $W$ boson mass, as well as a comparison of the resulting indirect constraints on the mass of the standard model Higgs boson and the direct measurements of the mass of the new boson observed at the LHC.
\end{abstract}

36th International Conference on High Energy Physics

4-11 July 2012

Melbourne, Australia

*Speaker.

$\dagger$ On behalf of the DØ Collaboration. 


\section{Introduction}

The standard model (SM) predicts a relation between the masses of the $W$ boson, the top quark and the Higgs boson. This relation arises from radiative corrections to the mass of the $W$ boson $\left(M_{W}\right)$, which include loops that contain top quarks, as well as Higgs boson loops. Extensions to the standard model, e.g. supersymmetry, predict new heavy particles that give rise to additional radiative (loop) corrections to the $W$ boson mass. Precise measurements of both the $W$ boson mass and the top quark mass can thus be used to predict, in the context of the SM, the mass of the Higgs boson. Together with the observation of a new boson at the LHC [1], these indirect constraints offer an important test of the SM: the direct measurements of the mass of the new boson can be compared to the indirect constraints to check if the SM relation between the three masses cited above is indeed verified, i.e. to check "if the new boson has the right mass to be indeed the Higgs boson of the SM". The precision of the indirect constraints is currently limited by the experimental precision on the $W$ boson mass. More precise measurements of $M_{W}$ are therefore an important contribution to our understanding of the electroweak interaction, and, potentially, of how the electroweak symmetry is broken. We present a new precision measurement of $M_{W}$ based on $4.3 \mathrm{fb}^{-1}$ of $\mathrm{D} \varnothing$ data, the new world average for $M_{W}$, as well as the latest comparison of indirect constraints on the Higgs boson mass and the direct measurements of the mass of the new boson observed at the LHC. A more detailed discussion of the importance of measurements of the $W$ boson mass, as well as of the measurement techniques used at the Tevatron can be found in Ref. [2].

\section{New measurement of the $W$ boson mass using $4.3 \mathrm{fb}^{-1}$ of DØ data}

The DØ Collaboration has recently published a new precision measurement of $M_{W}$ based on $4.3 \mathrm{fb}^{-1}$ of data [3]. This new measurement makes use of the methods that have been developed for an earlier measurement [4] based on an smaller, independent data set $\left(1 \mathrm{fb}^{-1}\right)$. Various parts of our model of the detector response have been refined to address the challenges posed by the high instantaneous luminosity of the larger data set. We use the channel $W \rightarrow e v$ and restrict ourselves to electrons reconstructed in the central calorimeter. In $4.3 \mathrm{fb}^{-1}$ of data, we select $1677394 \mathrm{~W} \rightarrow \mathrm{ev}$ candidate events. Since we are aiming at a relative uncertainty in $M_{W}$ of a few $10^{-4}$, we need to know the energy response for electrons from $W$ decay with a relative precision of a few $10^{-4}$. This stringent requirement can be met thanks to the powerful data control sample of $Z \rightarrow e^{+} e^{-}$ events and a detailed understanding of the response of the calorimeter to electromagnetic showers. We select 54512 candidate $Z \rightarrow e e$ events with both electrons in the central calorimeter. The dielectron invariant mass spectrum of these events is shown in Fig. 1. Also shown is the prediction of a simulation. The simulation combines parameterised models of different aspects of the detector response to electrons and to the hadrons recoiling against the $W$ or $Z$ boson (e.g. a parameterised model of the calorimeter energy response to the hadronic recoil) and predictions obtained from detailed first-principles simulations of the $\mathrm{D} \emptyset$ detector (e.g. simulations of the energy loss in the uninstrumented material in front of the calorimeter, and of sampling fluctuations in the calorimeter). The $Z \rightarrow e^{+} e^{-}$sample is critical for the tuning and validation of both the parameterisations and the first-principles simulation. It is also used, together with the precise measurements of the $Z$ boson mass from LEP, to determine the absolute energy scale of the calorimeter. 


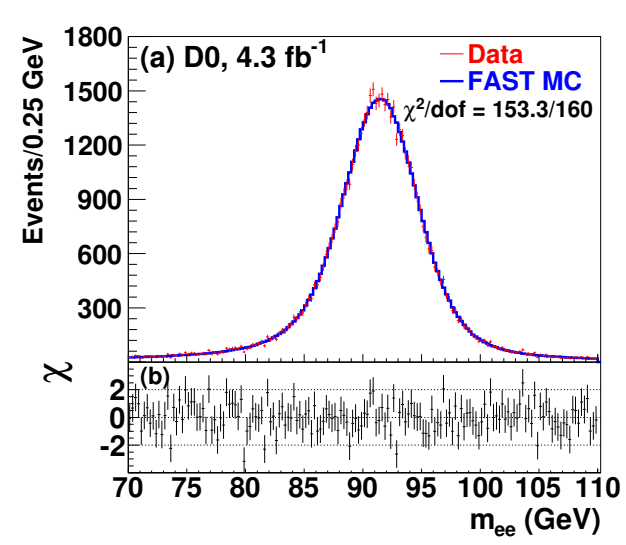

Figure 1: (a) Distribution of the di-electron invariant mass in the $Z \rightarrow e^{+} e^{-}$control sample. The shape of the prediction from the simulation ("FAST MC"), normalised to the data, is superimposed. (b) Bin-bybin difference between data and prediction, divided by the statistical uncertainty in the data.

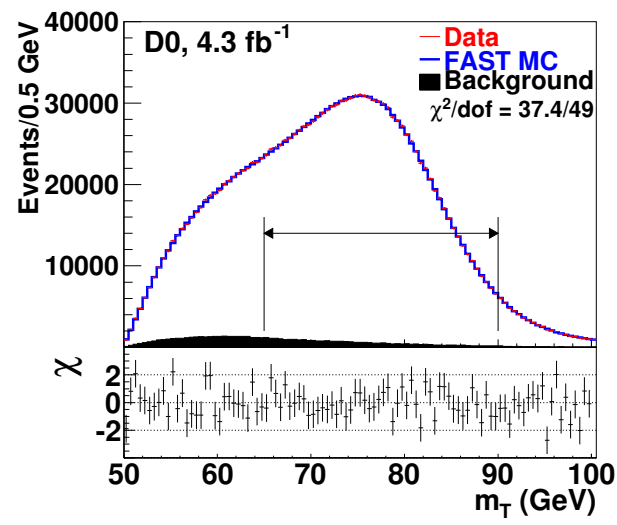

Figure 2: Distribution of the transverse mass in the $W \rightarrow e v$ data sample. The shape of the prediction from the simulation ("FAST MC"), normalised to the data, is superimposed. The value of $M_{W}$ that is input to the simulation has been adjusted to obtain the best agreement between simulation and data. The region of the distribution that is taken into account in this adjustment is indicated by the arrows.

The most powerful observable that is used to extract the $W$ boson mass is the so-called transverse mass $\left(m_{T}\right)$. It is defined as $m_{T}=\sqrt{2 p_{T}^{e} p_{T}^{v}(1-\cos \Delta \phi)}$, where $p_{T}^{e}$ is the transverse momentum of the electron, $\Delta \phi$ is the opening angle between the electron and neutrino momenta in the plane transverse to the beam. The vector $\vec{p}_{T}^{e}$ is determined using the energy measurement from the calorimeter and the direction measured in the central tracker. The vector $\vec{p}_{T}^{v}$ is equal to the event missing transverse momentum $\left(\vec{H}_{T}\right)$. The $m_{T}$ distribution in data is shown in Fig. 2. The procedure for the extraction of $M_{W}$ from this distribution is discussed in the caption of Fig. 2. The systematic uncertainties in the measurements of $M_{W}$ using three different observables $-m_{T}, p_{T}^{e}$ and $\not_{T}$ (and the same sample of $W$ candidate events) - are summarised in Fig. 3. The experimental uncertainties are dominated by the electron energy scale. This uncertainty, and most of the other experimental uncertainties, are driven by the limited $Z$ statistics. The largest source of uncertainty in the model of $W$ production and decay is the finite precision of the parton density functions (PDFs).

The results from the three observables are strongly correlated, albeit not fully. We use the methods from Ref. [5] to combine them. The result from $\not_{T}$ does not have any significant weight in the combination. A combination of the results from the $m_{T}$ and $p_{T}^{e}$ observables only yields $M_{W}=$ $80.367 \pm 0.013$ (stat.) \pm 0.022 (syst.) $\mathrm{GeV}=80.367 \pm 0.026 \mathrm{GeV}$. We further combine this result with the earlier measurement from Ref. [4] to obtain the new DØ Run II result: $M_{W}=80.375 \pm$ 0.011 (stat.) \pm 0.020 (syst.) $\mathrm{GeV}=80.375 \pm 0.023 \mathrm{GeV}$.

\section{New Tevatron and world averages}

Fig. 4 summarises all available measurements of $M_{W}$ from the Tevatron. These include the new result from $D \emptyset$ which was discussed above, as well as a new measurement [6] by the CDF Collaboration which has been published at about the same time. The techniques used by the two 


\begin{tabular}{lccc} 
& \multicolumn{3}{c}{$\Delta M_{W}(\mathrm{MeV})$} \\
Source & $m_{T}$ & $p_{T}^{e}$ & $B_{T}$ \\
\hline \hline Electron energy calibration & 16 & 17 & 16 \\
Electron resolution model & 2 & 2 & 3 \\
Electron shower modeling & 4 & 6 & 7 \\
Electron energy loss model & 4 & 4 & 4 \\
Hadronic recoil model & 5 & 6 & 14 \\
Electron efficiencies & 1 & 3 & 5 \\
Backgrounds & 2 & 2 & 2 \\
\hline Experimental subtotal & 18 & 20 & 24 \\
\hline PDF & 11 & 11 & 14 \\
QED & 7 & 7 & 9 \\
Boson $p_{T}$ & 2 & 5 & 2 \\
\hline Production subtotal & 13 & 14 & 17 \\
\hline Total & 22 & 24 & 29
\end{tabular}

Figure 3: Systematic uncertainties in the $M_{W}$ measurement using $4.3 \mathrm{fb}^{-1}$ of $\mathrm{D} \varnothing$ data.

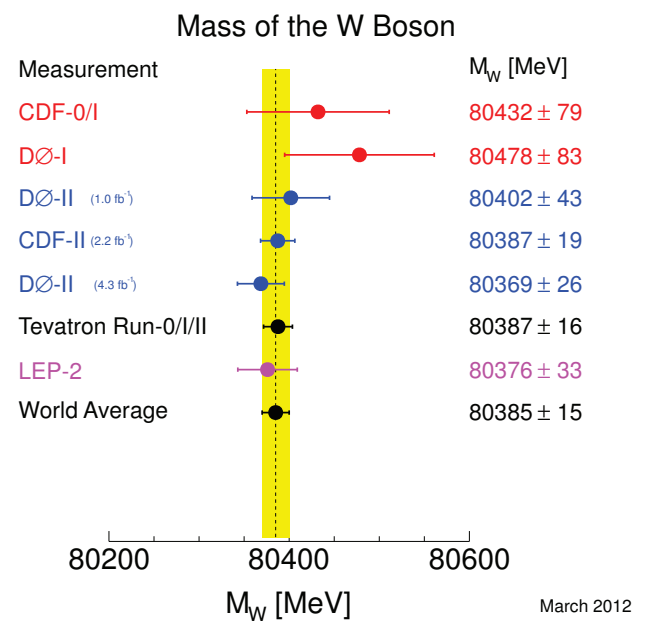

Figure 4: Summary of direct measurements of the $W$ boson mass. The results from Runs 0 and I of the Tevatron are shown in red, the latest results from Run II are shown in blue. These results are combined to obtain the Tevatron average. The combination of all results from LEP-2 is also included. The combination of the results from the Tevatron and LEP-2 yields the new world average.

collaborations to determine the single most important ingredient of this measurement, namely the lepton energy scale, are very different. As alluded to above, the DØ measurement relies on a detailed understanding of electron showers in the calorimeter, whereas the CDF measurement exploits precise measurements of track momenta in the inner detector. The results from the two experiments are of comparable precision, and the measurements are in good agreement. All results from the Tevatron are combined, again using the methods from Ref. [5], to yield the new Tevatron average for $M_{W}$ [7]. The new Tevatron average has an uncertainty of $16 \mathrm{MeV}$, which is considerably more precise than the combined result from the LEP experiments (with an uncertainty of $33 \mathrm{MeV}$ ) [8]. The new world average (LEP+Tevatron) has an uncertainty of $15 \mathrm{MeV}$ [7]. These averages are summarised in Fig. 4.

\section{Comparison of direct and indirect constraints on the Higgs boson mass}

Fits of the standard model of the electroweak interaction are a powerful tool for the comparison of direct and indirect constraints on the Higgs boson mass. A recent fit has been performed in Ref. [9]. In this fit, the free parameters of the SM, including the masses of the $W$ boson, the top quark and the Higgs boson, among others, are fit to the available experimental data. Thanks to the wealth of measurements of different observables that are available, this fit is overconstrained. This makes it possible to perform variants of the fit in which the direct measurements of one or more parameter are removed from the inputs to the fit. The fit then returns a prediction for the values of these parameters, based on the SM and the other experimental inputs. One such variant of the SM fit is shown in Fig. 5. This variant allows a comparison of the direct measurements of the masses of the $W$ boson and the top quark with indirect constraints from internal consistency of the SM. 


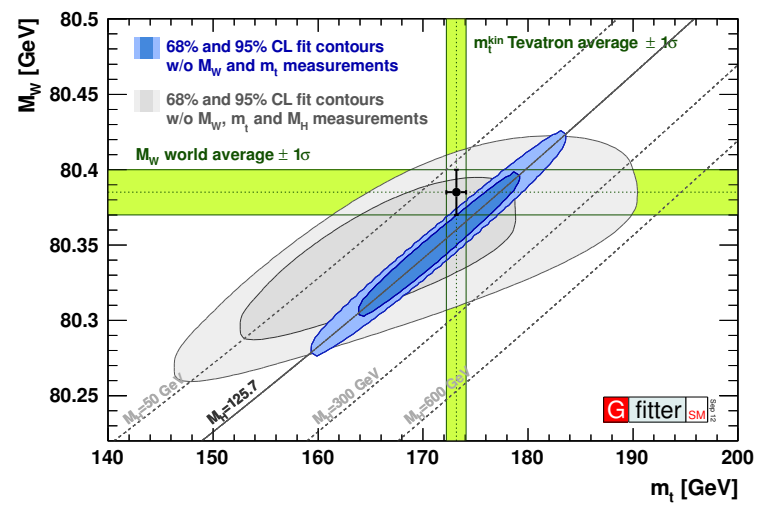

Figure 5: Indirect prediction for the masses of the top quark and the $W$ boson from internal consistency of the SM (labelled "68\% and 95\% CL fit contours w/o $M_{W}$ and $m_{t}$ measurements") [9]. This prediction uses the direct measurements of the mass of the candidate Higgs boson that has been discovered at the LHC [1]. The direct measurements of the $W$ boson mass (the world average discussed in Sec. 3 of the present article) and the Tevatron average of direct measurements of the top quark mass [10] are also shown.

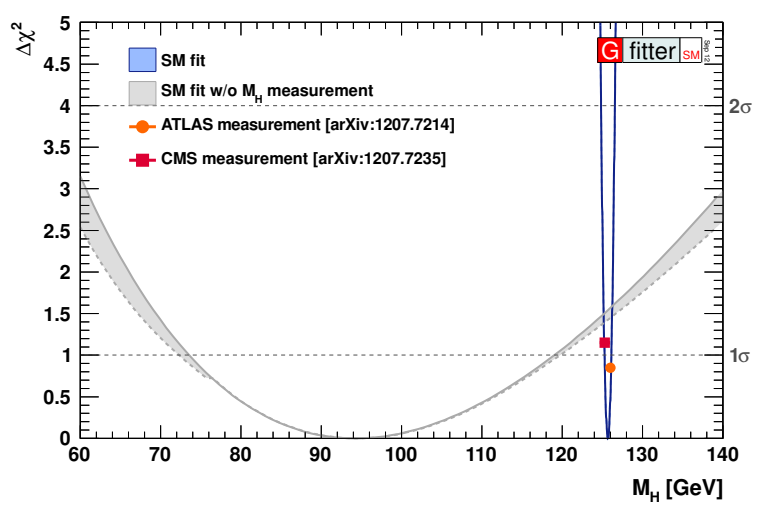

Figure 6: Indirect prediction for the mass of the Higgs boson from internal consistency of the SM (labelled "SM fit w/o $M_{H}$ measurement"). This prediction uses the new world average for $M_{W}$ which is discussed in Sec. 3 of the present article. The direct measurements of the mass of the new boson discovered [1] by the ATLAS and CMS experiments are also shown. The mass of the new boson is in agreement $(1.2 \sigma)$ with the indirect SM prediction for the Higgs boson mass.

Another variant, which allows for the most direct comparison of the direct and indirect constraints on the Higgs boson mass, is shown in Fig. 6. The indirect constraints prefer a Higgs boson that is somewhat lighter than the new boson discovered at the LHC, but they are still consistent, at the $1.2 \sigma$ level, with the mass of the new boson. This is an important success for the standard model.

\section{Conclusion and outlook}

We have reported a new precision measurement of the $W$ boson mass using $4.3 \mathrm{fb}^{-1}$ of $\mathrm{D} \varnothing$ data. The combination of this measurement and an earlier measurement using an independent $D \varnothing$ 
data set of $1 \mathrm{fb}^{-1}$ has an uncertainty of $23 \mathrm{MeV}$. Together with a new measurement by CDF, this improves the precision of the Tevatron average to $16 \mathrm{MeV}$. This is significantly more precise than the LEP average with its $33 \mathrm{MeV}$ uncertainty. The uncertainty of the new world average is $15 \mathrm{MeV}$. These improvements in the experimental precision on $M_{W}$ lead to precise indirect constraints on the mass of the SM Higgs boson. The direct measurements of the mass of the new boson discovered at the LHC agree, at the $1.2 \sigma$ level, with the indirect constraints. This is a remarkable success for the standard model. The limiting factor in the precision of this comparison of direct and indirect constraints is still the experimental precision on $M_{W}$. Even more precise measurements of $M_{W}$ are therefore needed to tighten the indirect constraints and make this test of the SM even more stringent. They will answer the question if the small $(1.2 \sigma)$ difference between direct and indirect constraints on the Higgs boson mass is a fluctuation or a first hint at physics beyond the standard model. The precision of the $\mathrm{D} \emptyset$ result is expected to improve significantly once the full data set (almost $10 \mathrm{fb}^{-1}$ ) has been analysed and once the end cap calorimeters are used to extend the electron coverage (which strongly reduces the sensitivity to the PDFs).

\section{References}

[1] G. Aad et al. (ATLAS Collaboration), "Observation of a new particle in the search for the standard model Higgs boson with the ATLAS detector at the LHC", Phys. Lett. B716, 1 (2012); S. Chatrchyan et al. (CMS Collaboration), "Observation of a new boson at a mass of $125 \mathrm{GeV}$ with the CMS experiment at the LHC”, Phys. Lett. B716, 30 (2012).

[2] A.V. Kotwal and J. Stark, "Measurement of the W boson mass at the Tevatron", Annu. Rev. Nucl. Part. Sci. 58, 147 (2008).

[3] V.M. Abazov et al. (DØ Collaboration), "Measurement of the $W$ boson mass with the D $\emptyset$ detector", Phys. Rev. Lett. 108, 151804 (2012).

[4] V.M. Abazov et al. (DØ Collaboration), “Measurement of the W boson mass", Phys. Rev. Lett. 103, 141801 (2009).

[5] L. Lyons, D. Gibout and P. Clifford, "How to combine correlated estimates of a single physical quantity", Nucl. Instrum. Methods Phys. Res., Sect. A270, 110 (1988); A. Valassi, "Combining correlated measurements of several different physical quantities", Nucl. Instrum. Methods Phys. Res., Sect. A500, 391 (2003).

[6] T. Aaltonen et al. (CDF Collaboration), "Precise measurement of the W boson mass with the CDF II detector", Phys. Rev. Lett. 108, 151803 (2012).

[7] The Tevatron electroweak working group for the CDF and D $\varnothing$ Collaborations, "2012 update of the combination of CDF and DØ results for the mass of the W boson", FERMI LAB-TM-2532-E, arXiv: 1204.0042 (2012)

[8] The LEP Collaborations: ALEPH, DELPHI, L3, OPAL and the LEP electroweak working group, "A combination of preliminary electroweak measurements and constraints on the standard model", arXiv: hepex/0612034 (2006).

[9] M. Baak et al. (Gfitter Group), "The electroweak fit of the standard model after the discovery of a new boson at the LHC”, Eur. Phys. J. C72 2205 (2012).

[10] T. Aaltonen et al. (CDF and DØ Collaborations), "Combination of the top quark mass measurements from the Tevatron collider”, Phys. Rev. D86, 092003 (2012). 